International Journal of Current Microbiology and Applied Sciences

ISSN: 2319-7706 Volume 10 Number 02 (2021)

Journal homepage: http://www.ijcmas.com

Original Research Article

https://doi.org/10.20546/ijcmas.2021.1002.183

\title{
Heterosis Studies in Muskmelon (Cucumis melo L.) for Yield and Quality Traits
}

\author{
Shivaji Kallappa Duradundi*, V. D. Gasti, Ravindra Mulage and \\ Deelipkumar A. Masuthi
}

Kittur Rani Channamma College of Horticulture, Arabhavi, India University of Horticultural Sciences, Bagalkot, India

*Corresponding author

\section{A B S T R A C T}

Keywords

Heterosis, Muskmelon

Article Info

Accepted:

15 January 2021

Available Online:

10 February 2021
An investigation was undertaken to study the heterosis in muskmelon (Cucumis melo L.) for yield and quality traits, during 2016-2017 at department of vegetable science, K. R. C. College of horticulture, Arabhavi. The ten lines and three testers were sown and crossed in a line $\mathrm{X}$ tester mating system to obtain $30 \mathrm{~F}_{1}$ hybrid combinations. Heterosis was valued in this study. Heterosis values were significant over better parent, best parent and over the commercial check in desirable direction in most crosses for yield and quality characters under studies. The cross involving KM-2 X PS exhibited maximum positive and significant heterosis over commercial check $(56.96 \%)$ for fruit yield per vine and fruit yield per hacter in muskmelon.

\section{Introduction}

The commercial exploitation of hybrid vigour depends on the ease with which the techniques are employed for hybrid seed production with minimum cost of seed production. Monoecious and romonoecious sex expression in muskmelon can be profitably utilised for the production of $F_{1}$ hybrid seeds at low cost where higher seed yield per pollination is observed. Monoecious lines used as female parents proved worthy for exploitation of heterosis observed for earliness, yield and yield component traits. Time required for pollination of given number of flowers is reduced by 50 per cent and fruit set percentage is high in monoecious varieties compared to and romonoecious varieties (Kesavan and More, 1991). Because of its wider spacing, the seed requirement per hectare for commercial hybrid cultivation would be low and cost effective. Therefore, muskmelon offers greater scope for the exploitation of hybrid vigour on commercial scale to increase the productivity 
and production. Intervarietal crosses of muskmelon recorded the marked heterosis for characters, such as the earliness, fruit weight and total yield per vine (Singh et al., 1976 and Munshi and Verma, 1997). Lal and Singh (1997) studied the genetic variability in 51 genotypes of muskmelon and genotype showed the high genetic variability for earliness, vine length, yield and quality characters except for number of fruits per vine. As the efforts in heterosis breeding are inadequate, the area under $F_{1}$ hybrids in muskmelon is very negligible in India. Most essential step in this direction is identification of superior heterotic $F_{1}$ hybrids for yield, quality and earliness. General and specific combing ability for quantitative characters influencing yield and its components is very helpful in selecting parents for production of superior hybrids. Several biometrical methods are available for studying the combining ability, heterosis and gene action. The line $\mathrm{x}$ tester $(1 \times t)$ analysis is one of the most used methods to test the large number of lines for combing ability and heterosis. With these backdrops, an effort to exploit heterosis in muskmelon was made in the present investigation through line $\mathrm{x}$ tester mating design with the objective of analysis of magnitude and direction of heterosis for yield and quality traits.

Muskmelon (Cucumis melo L.) $(2 \mathrm{n}=24)$ belongs to the family cucurbitaceae. Edible melons belong to either Cucumis melo var. reticulatus or Cucumis melo var. cantaloupensis. Plants are either monoecious or and romonoecious annuals with long trailing vines with shallow lobed round leaves. There is considerable variation in fruit size and shape. External appearance may be smooth with netted, the skin colour may be white, green and yellow. Yellowish brown or speckles yellow or orange with green or yellow background. Fruits of some cultivars crack when ripe. Upon ripening, fruits soften and fruity aromatic essence is formed in the fruit.

Muskmelon is used as dessert fruit and fruit juice has cooling effect. At greener stage, it is used as cooked vegetable. The fruit juice is nutritive and acts as demulcent and diuretic drink. Juice is also acts as remedy for skin diseases, tan freckles and dyspepsia. The seeds are edible and its kernel is rich in oil (40$44 \%$ ). This oil is useful in overcoming the problems like painful discharge and suppression of urine. The roots of melon have purgative and vomit causing properties. Fruits are good source of vitamins and minerals and relatively low in protein. The yellow and orange fleshed melons contain $\beta$-carotene and particularly cantaloupes are high in provitamin A (4200 IU/100 g). Melons are also rich in vitamin C (26 mg/100 g edible portion). For every $100 \mathrm{~g}$ edible portion, melons provide 26 to 17 calories energy, $0.3 \mathrm{~g}$ protein, $32 \mathrm{mg}$ calcium, $1.4 \mathrm{mg}$ iron and $14 \mathrm{mg}$ phosphorus (Chakrabarti, 2011).

\section{Materials and Methods}

The experiment on heterosis studies in muskmelon (Cucumis melo L.) was conducted in the fields of Vegetable Science unit of Kittur Rani Channamma College of Horticulture, Arabhavi, Belagavi District (Karnataka). Seeds of lines and testers were sown during the month of June 2016 for attempting crosses in line $\mathrm{x}$ tester fashion. Sowing was done on $2.00 \mathrm{~m}$ apart ridges at a spacing of $0.90 \mathrm{~m}$ between plants for easy movement. All the recommended cultivation practices were followed to raise a good crop (as per the package of practices of horticultural crops of University of Horticultural Sciences, Bagalkot (Anon., 2014). A total of 30 hybrids were developed by crossing 10 female parents (lines) with each of three male parents (testers). Flower buds of male and female parents were selected 
on the previous evening prior to the day of their opening. The selected flower buds of male parents were covered with butter paper bags to avoid contamination of pollens by other parents. Similarly flower buds of female parents were emasculated and covered with butter paper bag to avoid out crossing. Pollination was carried out on the next day morning between 5.30 am and 8.00 am by using pollens of desired male parents. After pollination, the female flower buds were again covered with butter paper bags to avoid contamination and tagged with the details of male parent and date of pollination. Simultaneously, the male and female parents were selfed by bagging the flower buds with butter paper bags prior to the day of flower opening. Crossed and selfed fruits were harvested separately at full maturity stage. The seeds were hand extracted and preserved in butter paper bags labelled with the details of cross or entry number. The mean of all the replications for each parents, hybrids and check for each of the characters was computed and used in estimation of heterosis. Heterosis was calculated as the percentage increase or decrease of mean $F_{1}$ performance $\left(F_{1}\right)$ over the means of better parent (BP), the best parent (BTP) and the commercial check (CC). Replication means of various characters of parents and hybrids were subjected to line $\mathrm{x}$ tester $(l \times \mathrm{t})$ with randomised block design analysis (Kempthorne, 1957).

\section{Results and Discussion}

Results of analysis of variance for 14 characters under study are summarised in table 1 and variance of 30 hybrids and 13 parents showed that mean square due to all sources of variation for all the yield and quality traits were significantly different except testers. The main aim of any breeding programme is to enhance the yielding ability of the crop. Heterosis breeding offers quick and quantum jump in yield. $\mathrm{F}_{1}$ hybrids derived from crossing of pure lines are exceptionally uniform in growth and development and possess better quality and adaptability to varied environmental conditions and give high early and total yields and can be exploited in rapid deployment of dominant genes for resistance to diseases and pests (Riggs, 1988). Hence for exploitation of heterosis, choice of suitable parents is an important pre-requisite. In practical plant breeding, superiority of the $\mathrm{F}_{1}$ over mid parent is of little value, since it does not offer any advantage to exploit it commercially. The commercial usefulness of the hybrid would depend on its performance in comparison to the best existing commercial variety or hybrid. Hence, heterosis over better parent, best parent and commercial check was worked out in the present investigation for identification of superior hybrids.

There was highly significant difference among genotypes for all the yield and quality parameters under studied (Table 1). Genetic diversity among parents can ensure higher magnitude of heterosis and mean sum of squares of genotypes were found to be highly significant for all studied traits, this provides evidence for presence of considerable amount of genetic variation among the genotypes. These results are in same line with those obtained by El-shimi et al., (2003), Hatem et al., (2009) and Bayoumy et al., (2014) in melon.

Ultimate goal of the plant breeder is to develop high yielding varieties or hybrids. The yield components greatly influence yield and higher magnitude of heterosis was observed for the yield components in the present investigation. Number of fruiting branches per vine, number of fruits per vine and average fruit weight can greatly contribute for heterosis observed for total fruit yield per vine are very closely related to productivity parameters. For all these traits positive heterosis is desirable. The highest standard 
heterosis was observed in the cross KM-2 $\mathrm{x}$ PS $(38.60 \%)$ (Table 2) for number of fruiting branches per vine, which is of higher magnitude and direction of that of reports from Vishwanatha (2003). The cross KM-2 x PS $(53.13 \%)$ (Table 2) expressed maximum standard heterosis for number of fruits per vine and the magnitude of heterosis is of similar to that of Choudhary et al., (2003) and it was higher heterosis per cent as compared to report of Subramanian (2008). The cross KM1 x HM (52.79 \%) (Table 2) shown maximum standard heterosis for average fruit weight and is comparable with that reports by Dhaliwal and Lal (1996), Shashikumar and Pitchaimuthu (2016) and it was lower than that of reports of Subramanian (2008).

The magnitude of heterosis over better parent, best parent and commercial check was highly significant for fruit yield per vine. Out of the 30 crosses, 21 crosses over better parent, 20 crosses over best parent and 16 crosses over commercial check exhibited positive and significant heterosis for fruit yield per vine (Table 3).

The maximum heterosis over commercial check was observed in the cross KM-2 x PS (56.96 \%) followed by KM-1 x DK (52.17\%), KM-3 x PS (46.52\%), KM-10 x HM (43.48 $\%), \mathrm{KM}-10$ x PS (40.43\%), KM-10 x DK (37.83 \%), KM-8 x DK (35.22\%) and KM-1 x HM (32.17 \%) (Table 3). Magnitude of heterosis is comparable with the findings of Shashikumar and Pitchaimuthu (2016) and high in comparison with the findings of Vishwanatha (2003) and it is attributed to use of different genetic stocks and also varied environmental conditions.

Magnitude of heterosis over better parent, best parent and commercial check was highly significant in both the directions for fruit yield per plot. Out of the 30 crosses, 19 crosses over better parent, 16 crosses over best parent and
16 crosses over commercial check showed positive and significant heterosis for fruit yield per plot.

The maximum heterosis over commercial check was observed in the crosses KM-2 x PS (56.96 \%) followed by KM-1 x DK $(52.17 \%)$, KM-3 x PS (46.52\%), KM-10 x HM (43.48 $\%), \mathrm{KM}-10$ x PS (40.43\%), KM-10 x DK (37.83\%), KM-8 x DK (35.00 \%) and KM-1 x HM (32.17 \%) (Table 3). Magnitude of heterosis was low in comparison with the findings of Subramanian (2008) and high in comparison with the findings of Vishwanatha (2003) and it is attributed to use of different genetic stocks and also varied environmental conditions.

Magnitude of heterosis over better parent, best parent and commercial check was highly significant in both the directions for fruit yield per hectare. Out of the 30 crosses, 20 crosses over better parent, 18 crosses over best parent and 16 crosses over commercial check showed positive and significant heterosis for fruit yield per hectare.

The maximum heterosis over commercial check was observed in the cross KM-2 x PS (56.96 \%) followed by KM-1 x DK (52.19\%), KM-3 x PS (46.56 \%), KM-10 x HM (43.51 $\%), \mathrm{KM}-10$ x PS (40.45 \%), KM-10 x DK (37.87 \%), KM-8 x DK (34.98 \%) and KM-1 x HM (32.16 \%) (Table 3). Magnitude of heterosis is comparable with the findings of Shashikumar and Pitchaimuthu (2016) and high in comparison with the findings of Vishwanatha (2003).

Fifteen crosses exhibited significant standard heterosis in desirable direction and maximum was observed in the cross KM-8 x PS (-16.22 $\%$ ) (Table 4) for fruit shape index which was slightly lesser than that of reports by Vishwanatha (2003). 
Table.1 Analysis of variance (mean sum of squares) of line $\mathrm{x}$ tester analysis for various characters in muskmelon

\begin{tabular}{|c|c|c|c|c|c|c|c|c|c|c|}
\hline $\begin{array}{l}\text { Sl. } \\
\text { No. }\end{array}$ & Character & $\begin{array}{l}\text { Replicatio } \\
\text { ns }\end{array}$ & Genotypes & Parents & $\begin{array}{l}\text { Parents vs } \\
\text { Crosses }\end{array}$ & Crosses & Lines & Testers & $\begin{array}{l}\text { Line } \mathbf{x} \\
\text { Tester }\end{array}$ & Error \\
\hline & Degrees of freedom & 1 & 42 & 12 & 1 & 29 & 9 & 2 & 18 & 42 \\
\hline $\mathbf{a}$ & Yield and yield parameters & & & & & & & & & \\
\hline 1 & $\begin{array}{c}\text { Number of fruiting branches per } \\
\text { vine }\end{array}$ & $2.376^{\mathrm{NS}}$ & $16.853 * *$ & $3.592 * *$ & $313.181 * *$ & $12.123 * *$ & $20.922 *$ & $7.285^{\mathrm{NS}}$ & $8.261 * *$ & 1.880 \\
\hline 2 & Number of fruits per vine & $0.079^{\mathrm{NS}}$ & $1.374 * *$ & $0.217 * *$ & $15.577 * *$ & $1.363 * *$ & $1.625^{\mathrm{NS}}$ & $1.389^{\mathrm{NS}}$ & $1.229 * *$ & 0.250 \\
\hline 3 & Average fruit weight (g) & $92.250^{\mathrm{NS}}$ & $49256.410 * *$ & $8537.367 * *$ & $479623.174 * *$ & $51265.43 * *$ & $53021.72^{\mathrm{NS}}$ & $11645.62^{\mathrm{NS}}$ & $54789.5^{* *}$ & 78.337 \\
\hline 4 & Fruit yield per vine $(\mathrm{kg})$ & $0.407^{\mathrm{NS}}$ & $0.803 * *$ & $0.512 * *$ & $7.812 * *$ & $0.867 * *$ & $1.601 *$ & $0.456^{\mathrm{NS}}$ & $0.545^{* *}$ & 0.149 \\
\hline 5 & Fruit yield per plot (kg) & $40.685^{\mathrm{NS}}$ & $80.268 * *$ & $50.012 * *$ & $781.220 * *$ & $86.657 * *$ & $160.125^{*}$ & $45.645^{\mathrm{NS}}$ & $54.480 * *$ & 14.877 \\
\hline 6 & Fruit yield per hectare $(\mathrm{t})$ & $8.916^{\mathrm{NS}}$ & $24.785 * *$ & $15.023 * *$ & $241.192 * *$ & $26.758 * *$ & $49.450 *$ & $14.105^{\mathrm{NS}}$ & $16.818 * *$ & 4.596 \\
\hline b & Quality parameters & & & & & & & & & \\
\hline 7 & Fruit shape index & $0.004^{\mathrm{NS}}$ & $0.073 * *$ & $0.018 * *$ & $0.667 * *$ & $0.075 * *$ & $0.109^{\mathrm{NS}}$ & $0.003^{\mathrm{NS}}$ & $0.065 * *$ & 0.012 \\
\hline 8 & Flesh thickness $(\mathrm{cm})$ & $0.006^{\mathrm{NS}}$ & $0.775^{* *}$ & $0.093 * *$ & $7.070 * *$ & $0.840 * *$ & $0.540^{\mathrm{NS}}$ & $0.230^{\mathrm{NS}}$ & $1.058 * *$ & 0.065 \\
\hline 9 & Rind thickness $(\mathrm{cm})$ & $0.000^{\mathrm{NS}}$ & $0.009 * *$ & $0.00092^{\mathrm{NS}}$ & $0.034 * *$ & $0.012 * *$ & $0.013^{\mathrm{NS}}$ & $0.004^{\mathrm{NS}}$ & $0.012 * *$ & 0.003 \\
\hline 10 & Cavity length $(\mathrm{cm})$ & $1.001^{\mathrm{NS}}$ & $1.718 * *$ & $1.303 * *$ & $7.385 * *$ & $2.108 * *$ & $0.820^{\mathrm{NS}}$ & $1.997^{\mathrm{NS}}$ & $2.764 * *$ & 0.407 \\
\hline 11 & Cavity breadth $(\mathrm{cm})$ & $0.022^{\mathrm{NS}}$ & $1.999 * *$ & $0.962 * *$ & $11.714 * *$ & $2.093 * *$ & $2.406^{\mathrm{NS}}$ & $0.821^{\mathrm{NS}}$ & $2.078 * *$ & 0.347 \\
\hline 12 & Total soluble solids ( $\mathrm{Brix}$ ) & $0.046^{\mathrm{NS}}$ & $7.472 * *$ & $1.636^{* *}$ & $38.886^{* *}$ & $8.804^{* *}$ & $15.836^{*}$ & $2.441^{\mathrm{NS}}$ & $5.995 * *$ & 0.611 \\
\hline 13 & Total sugars (\%) & $0.426^{\mathrm{NS}}$ & $4.093 * *$ & $0.564 * *$ & $15.723 * *$ & $5.153 * *$ & $8.841^{\mathrm{NS}}$ & $0.672^{\mathrm{NS}}$ & $3.806^{* *}$ & 0.347 \\
\hline 14 & $\beta$-carotene content $(\mu \mathrm{g} / 100 \mathrm{~g})$ & $325.963^{\mathrm{NS}}$ & $28863.716^{* *}$ & $3194.303 * *$ & $110196.574 * *$ & $36680.96 * *$ & $63004.01 *$ & $29687.85^{\mathrm{NS}}$ & $24296.4 * *$ & 157.705 \\
\hline
\end{tabular}

$*$ and $* *$ indicate significance of values at $\mathrm{p}=0.05$ and $\mathrm{p}=0.01$, respectively. NS: Non significant, DAS: Days after sowing 
Table.2 Heterosis (\%) over better parent, best parent and commercial check for number of fruiting branches per vine, number of fruits per vine and average fruit weight in muskmelon

\begin{tabular}{|c|c|c|c|c|c|c|c|c|c|c|}
\hline \multirow[t]{2}{*}{ Sl. No. } & \multirow[t]{2}{*}{ Crosses } & \multicolumn{3}{|c|}{$\begin{array}{l}\text { Number of fruiting branches per } \\
\text { vine }\end{array}$} & \multicolumn{3}{|c|}{ Number of fruits per vine } & \multicolumn{3}{|c|}{ Average fruit weight } \\
\hline & & BP & BTP & $\mathrm{CC}$ & BP & BTP & $\mathrm{CC}$ & BP & BTP & $\mathrm{CC}$ \\
\hline 1 & $\mathrm{KM}-1 \times \mathrm{PS}$ & $23.31 * *$ & $18.93 * *$ & $17.54 * *$ & $20.69 * *$ & $12.90 * *$ & $9.38 * *$ & $33.00 * *$ & $20.36 *$ & $23.38 *$ \\
\hline 2 & $\mathrm{KM}-1 \times \mathrm{HM}$ & $29.45 * *$ & $24.85 * *$ & $23.39 * *$ & $51.85 * *$ & $32.26 * *$ & $28.13 * *$ & $68.24 * *$ & $49.05 * *$ & $52.79 * *$ \\
\hline 3 & $\mathrm{KM}-1 \times \mathrm{DK}$ & $41.10 * *$ & $36.09 * *$ & $34.50 * *$ & $66.67 * *$ & $45.16^{* *}$ & $40.63 * *$ & $38.64 * *$ & $23.53 *$ & $26.63 * *$ \\
\hline 4 & $\mathrm{KM}-2 \times \mathrm{PS}$ & $40.24 * *$ & $40.24 * *$ & $38.60 * *$ & $58.06^{* *}$ & $58.06 * *$ & $53.13 * *$ & $22.02 *$ & $22.02 *$ & $25.08 * *$ \\
\hline 5 & $\mathrm{KM}-2 \times \mathrm{HM}$ & $21.30 * *$ & $21.30 * *$ & $19.88 * *$ & $6.45 * *$ & $6.45 * *$ & $3.13 * *$ & 7.49 & 7.49 & 10.19 \\
\hline 6 & $\mathrm{KM}-2 \times \mathrm{DK}$ & $13.02 * *$ & $13.02 * *$ & $11.70 * *$ & $9.68 * *$ & $9.68 * *$ & $6.25^{* *}$ & 12.03 & 12.03 & 14.84 \\
\hline 7 & KM-3 × PS & $42.31 * *$ & $31.36 * *$ & $29.82 * *$ & $55.17 * *$ & $45.16 * *$ & $40.63 * *$ & $44.53 * *$ & $34.37 * *$ & $37.75 * *$ \\
\hline 8 & $\mathrm{KM}-3 \times \mathrm{HM}$ & $16.03 * *$ & $7.10 * *$ & $5.85^{* *}$ & $29.63 * *$ & $12.90 * *$ & $9.38 * *$ & $27.57 * *$ & $18.60 *$ & $21.58 *$ \\
\hline 9 & $\mathrm{KM}-3 \times \mathrm{DK}$ & $3.85 * *$ & $-4.14 * *$ & $-5.26 * *$ & $-37.04 * *$ & $-45.16 * *$ & $-46.88 * *$ & $-20.94 *$ & $-26.50 * *$ & $-24.65 * *$ \\
\hline 10 & $\mathrm{KM}-4 \times \mathrm{PS}$ & $26.80 * *$ & $14.79 * *$ & $13.45^{* *}$ & $41.38 * *$ & $32.26 * *$ & $28.13^{* *}$ & $43.86^{* *}$ & $30.19 * *$ & $33.46 * *$ \\
\hline 11 & $\mathrm{KM}-4 \times \mathrm{HM}$ & $12.50 * *$ & $-4.14 * *$ & $-5.26 * *$ & $-19.23 * *$ & $-32.26 * *$ & $-34.38 * *$ & 6.19 & -6.11 & -3.75 \\
\hline 12 & $\mathrm{KM}-4 \times \mathrm{DK}$ & $45.39 * *$ & $21.30 * *$ & $19.88 * *$ & $43.48 * *$ & $6.45 * *$ & $3.13 * *$ & $42.15 * *$ & $26.65 * *$ & $29.83 * *$ \\
\hline 13 & $\mathrm{KM}-5 \times \mathrm{PS}$ & $20.92 * *$ & $9.47 * *$ & $8.19 * *$ & 0.00 & $-6.45 * *$ & $-9.38 * *$ & $56.60 * *$ & $41.72 * *$ & $45.28 * *$ \\
\hline 14 & $\mathrm{KM}-5 \times \mathrm{HM}$ & $39.58 * *$ & $18.93 * *$ & $17.54 * *$ & $50.00 * *$ & $25.81 * *$ & $21.88 * *$ & -16.93 & $-26.55 * *$ & $-24.71 * *$ \\
\hline 15 & $\mathrm{KM}-5 \times \mathrm{DK}$ & $14.18 * *$ & $-4.73 * *$ & $-5.85^{* *}$ & $26.09 * *$ & $-6.45 * *$ & $-9.38 * *$ & $42.50 * *$ & $26.97 * *$ & $30.15 * *$ \\
\hline 16 & KM-6 × PS & $26.80 * *$ & $14.79 * *$ & $13.45^{* *}$ & $31.03 * *$ & $22.58 * *$ & $18.75^{* *}$ & $45.29 * *$ & $31.48 * *$ & $34.78 * *$ \\
\hline 17 & KM-6 × HM & $13.19 * *$ & $-3.56^{*}$ & $-4.68 * *$ & $-26.92 * *$ & $-38.71 * *$ & $-40.63 * *$ & 0.02 & -11.56 & -9.34 \\
\hline 18 & KM-6 × DK & $4.90 * *$ & $-11.24 * *$ & $-12.28 * *$ & 0.00 & $-25.81 * *$ & $-28.13 * *$ & 6.81 & -4.83 & -2.44 \\
\hline \multirow[t]{2}{*}{ Sl. No. } & \multirow[t]{2}{*}{ Crosses } & \multicolumn{3}{|c|}{$\begin{array}{c}\text { Number of fruiting branches per } \\
\text { vine }\end{array}$} & \multicolumn{3}{|c|}{ Number of fruits per vine } & \multicolumn{3}{|c|}{ Average fruit weight } \\
\hline & & $\mathbf{B P}$ & BTP & $\mathrm{CC}$ & BP & BTP & $\mathrm{CC}$ & BP & BTP & $\mathbf{C C}$ \\
\hline 19 & $\mathrm{KM}-7 \times \mathrm{PS}$ & $23.53 * *$ & $11.83 * *$ & $10.53 * *$ & $13.79 * *$ & $6.45 * *$ & $3.13 * *$ & $-26.76 * *$ & $-33.72 * *$ & $-32.06 * *$ \\
\hline 20 & $\mathrm{KM}-7 \times \mathrm{HM}$ & $9.03 * *$ & $-7.10 * *$ & $-8.19 * *$ & $11.54 * *$ & $-6.45 * *$ & $-9.38 * *$ & $48.44 * *$ & $31.25 * *$ & $34.54 * *$ \\
\hline 21 & $\mathrm{KM}-7 \times \mathrm{DK}$ & $36.17 * *$ & $13.61 * *$ & $12.28 * *$ & $52.17 * *$ & $12.90 * *$ & $9.38 * *$ & -8.85 & $-18.79 *$ & -16.75 \\
\hline
\end{tabular}


Int.J.Curr.Microbiol.App.Sci (2021) 10(02): 1534-1549

\begin{tabular}{|c|c|c|c|c|c|c|c|c|c|c|}
\hline 22 & $\mathrm{KM}-8 \times \mathrm{PS}$ & $11.73 * *$ & $7.10 * *$ & $5.85 * *$ & $17.24 * *$ & $9.68 * *$ & $6.25 * *$ & -15.52 & $-23.54 *$ & $-21.62 *$ \\
\hline 23 & $\mathrm{KM}-8 \times \mathrm{HM}$ & $26.54 * *$ & $21.30 * *$ & $19.88 * *$ & $46.15^{* *}$ & $22.58 * *$ & $18.75^{* *}$ & $48.97 * *$ & $32.53 * *$ & $35.86 * *$ \\
\hline 24 & $\mathrm{KM}-8 \times \mathrm{DK}$ & $25.31 * *$ & $20.12 * *$ & $18.71 * *$ & $64.00 * *$ & $32.26 * *$ & $28.13 * *$ & $45.48 * *$ & $29.63 * *$ & $32.88 * *$ \\
\hline 25 & KM-9 $\times$ PS & $-3.27 *$ & $-12.43 * *$ & $-13.45 * *$ & $-13.79 * *$ & $-19.35 * *$ & $-21.88 * *$ & 4.99 & -4.99 & -2.60 \\
\hline 26 & KM-9 × HM & 0.67 & $-11.24 * *$ & $-12.28 * *$ & $-11.54 * *$ & $-25.81 * *$ & $-28.13 * *$ & -8.62 & $-18.98 *$ & -16.94 \\
\hline 27 & $\mathrm{KM}-9 \times \mathrm{DK}$ & $22.82 * *$ & $8.28 * *$ & $7.02 * *$ & $48.00 * *$ & $19.35 * *$ & $15.63 * *$ & -14.97 & $-24.24 * *$ & $-22.33 *$ \\
\hline 28 & $\mathrm{KM}-10 \times \mathrm{PS}$ & $37.34 * *$ & $28.40 * *$ & $26.90 * *$ & $48.28 * *$ & $38.71 * *$ & $34.38 * *$ & $40.68 * *$ & $27.31 * *$ & $30.51 * *$ \\
\hline 29 & KM-10 × HM & $36.08 * *$ & $27.22 * *$ & $25.73 * *$ & $59.26 * *$ & $38.71 * *$ & $34.38 * *$ & $43.31 * *$ & $26.71 * *$ & $29.90 * *$ \\
\hline \multirow[t]{4}{*}{30} & $\mathrm{KM}-10 \times \mathrm{DK}$ & $32.91 * *$ & $24.26 * *$ & $22.81 * *$ & $51.85 * *$ & $32.26 * *$ & $28.13 * *$ & $43.77 * *$ & $28.10 * *$ & $31.32 * *$ \\
\hline & SEm \pm & 0.961 & 0.961 & 0.961 & 0.350 & 0.350 & 0.350 & 6.225 & 6.225 & 6.225 \\
\hline & $\mathrm{CD}$ at $5 \%$ & 2.74 & 2.74 & 2.74 & 1.00 & 1.00 & 1.00 & 17.75 & 17.75 & 17.75 \\
\hline & $\mathrm{CD}$ at $1 \%$ & 3.66 & 3.66 & 3.66 & 1.33 & 1.33 & 1.33 & 23.73 & 23.73 & 23.73 \\
\hline
\end{tabular}

*and** indicate significance of values at $\mathrm{p}=0.05$ and $\mathrm{p}=0.01$, respectively. BP, BTP and CC: Heterosis over better parent, best parent and commercial check (Madhuras),

respectively. PS: Punjab Sunheri HM: Hara Madhu DK: Durga Kranti 
Table.3 Heterosis (\%) over better parent, best parent and commercial check for fruit yield per vine, fruit yield per plot and fruit yield per hectare in muskmelon

\begin{tabular}{|c|c|c|c|c|c|c|c|c|c|c|}
\hline \multirow[t]{2}{*}{ Sl. No. } & \multirow[t]{2}{*}{ Crosses } & \multicolumn{3}{|c|}{ Fruit yield per vine } & \multicolumn{3}{|c|}{ Fruit yield per plot } & \multicolumn{3}{|c|}{ Fruit yield per hectare } \\
\hline & & BP & BTP & $\mathrm{CC}$ & BP & BTP & $\mathrm{CC}$ & BP & BTP & $\mathrm{CC}$ \\
\hline 1 & $\mathrm{KM}-1 \times \mathrm{PS}$ & $51.57 * *$ & $32.49 * *$ & $26.09 * *$ & $51.57 * *$ & $32.49 * *$ & $25.87 * *$ & $51.53 * *$ & $32.50 * *$ & $25.90 * *$ \\
\hline 2 & KM-1 $\times$ HM & $62.13 * *$ & $39.13 * *$ & $32.17 * *$ & $62.13 * *$ & $39.13 * *$ & $32.17 * *$ & $62.17 * *$ & $39.13 * *$ & $32.16 * *$ \\
\hline 3 & $\mathrm{KM}-1 \times \mathrm{DK}$ & $86.67 * *$ & $60.18 * *$ & $52.17 * *$ & $86.67 * *$ & $60.18 * *$ & $52.17 * *$ & $86.70 * *$ & $60.17 * *$ & $52.19 * *$ \\
\hline 4 & $\mathrm{KM}-2 \times \mathrm{PS}$ & $65.22 * *$ & $65.22 * *$ & $56.96 * *$ & $65.22 * *$ & $65.22 * *$ & $56.96 * *$ & $65.20 * *$ & $65.20 * *$ & $56.96 * *$ \\
\hline 5 & $\mathrm{KM}-2 \times \mathrm{HM}$ & $13.96 * *$ & $13.96 * *$ & $8.26 * *$ & $13.96 * *$ & $13.96 * *$ & $8.26^{*}$ & $13.92 * *$ & $13.92 * *$ & $8.22 * *$ \\
\hline 6 & $\mathrm{KM}-2 \times \mathrm{DK}$ & $4.81 * *$ & $4.81 * *$ & -0.43 & 4.81 & 4.81 & -0.43 & $4.82 *$ & $4.82 *$ & -0.39 \\
\hline 7 & $\mathrm{KM}-3 \times \mathrm{PS}$ & $76.44 * *$ & $54.23 * *$ & $46.52 * *$ & $76.44 * *$ & $54.23 * *$ & $46.52 * *$ & $76.40 * *$ & $54.24 * *$ & $46.56 * *$ \\
\hline 8 & $\mathrm{KM}-3 \times \mathrm{HM}$ & $23.10 * *$ & $3.66 * *$ & $-1.30 * *$ & $23.10 * *$ & 3.66 & -1.52 & $23.14 * *$ & 3.67 & -1.49 \\
\hline 9 & $\mathrm{KM}-3 \times \mathrm{DK}$ & $1.63 * *$ & $-14.65 * *$ & $-18.70 * *$ & 1.63 & $-14.65 * *$ & $-18.91 * *$ & 1.62 & $-14.66 * *$ & $-18.94 * *$ \\
\hline 10 & $\mathrm{KM}-4 \times \mathrm{PS}$ & $38.48 * *$ & $21.05 * *$ & $15.22 * *$ & $38.48 * *$ & $21.05 * *$ & $15.00 * *$ & $38.44 * *$ & $21.05 * *$ & $15.02 * *$ \\
\hline 11 & $\mathrm{KM}-4 \times \mathrm{HM}$ & $-8.42 * *$ & $-22.88 * *$ & $-26.52 * *$ & $-8.42 *$ & $-22.88 * *$ & $-26.74 * *$ & $-8.41 * *$ & $-22.90 * *$ & $-26.76 * *$ \\
\hline 12 & $\mathrm{KM}-4 \times \mathrm{DK}$ & $40.60 * *$ & $18.08 * *$ & $12.17 * *$ & $40.60 * *$ & $18.08 * *$ & $12.17 * *$ & $40.61 * *$ & $18.08 * *$ & $12.21 * *$ \\
\hline 13 & $\mathrm{KM}-5 \times \mathrm{PS}$ & $19.90 * *$ & $4.81 * *$ & -0.43 & $19.90 * *$ & 4.81 & -0.43 & $19.88 * *$ & $4.82 *$ & -0.39 \\
\hline 14 & KM-5 × HM & $38.59 * *$ & $16.70 * *$ & $10.87 * *$ & $38.59 * *$ & $16.70 * *$ & $10.87 * *$ & $38.60 * *$ & $16.68 * *$ & $10.88 * *$ \\
\hline 15 & $\mathrm{KM}-5 \times \mathrm{DK}$ & $38.96 * *$ & $16.70 * *$ & $10.87 * *$ & $38.96 * *$ & $16.70 * *$ & $10.87 * *$ & $38.94 * *$ & $16.68 * *$ & $10.88 * *$ \\
\hline 16 & KM-6 × PS & $49.48 * *$ & $30.66 * *$ & $24.35 * *$ & $49.48 * *$ & $30.66 * *$ & $24.13 * *$ & $49.41 * *$ & $30.64 * *$ & $24.10 * *$ \\
\hline 17 & KM-6 × HM & $-3.26 * *$ & $-18.54 * *$ & $-22.61 * *$ & -3.26 & $-18.54 * *$ & $-22.61 * *$ & -3.28 & $-18.57 * *$ & $-22.61 * *$ \\
\hline 18 & KM-6 × DK & $-7.08 * *$ & $-21.97 * *$ & $-25.65 * *$ & -7.08 & $-21.97 * *$ & $-25.87 * *$ & $-7.11 * *$ & $-21.99 * *$ & $-25.90 * *$ \\
\hline 19 & $\mathrm{KM}-7 \times \mathrm{PS}$ & $-16.75 * *$ & $-27.23 * *$ & $-30.87 * *$ & $-16.75 * *$ & $-27.23 * *$ & $-30.87 * *$ & $-16.77 * *$ & $-27.22 * *$ & $-30.83 * *$ \\
\hline \multirow[t]{2}{*}{ Sl. No. } & Crosses & \multicolumn{3}{|c|}{ Fruit yield per vine } & \multicolumn{3}{|c|}{ Fruit yield per plot } & \multicolumn{3}{|c|}{ Fruit yield per hectare } \\
\hline & & BP & BTP & $\mathbf{C C}$ & $\mathbf{B P}$ & BTP & $\mathbf{C C}$ & $\mathbf{B P}$ & BTP & $\mathbf{C C}$ \\
\hline 20 & $\mathrm{KM}-7 \times \mathrm{HM}$ & $-16.58 * *$ & $-29.75 * *$ & $-33.04 * *$ & $-16.58 * *$ & $-29.75 * *$ & $-33.26 * *$ & $-16.54 * *$ & $-29.74 * *$ & $-33.26 * *$ \\
\hline 21 & $\mathrm{KM}-7 \times \mathrm{DK}$ & $21.53 * *$ & $2.06 * *$ & $-3.04 * *$ & $21.53 * *$ & 2.06 & -3.04 & $21.48 * *$ & 2.02 & -3.05 \\
\hline 22 & $\mathrm{KM}-8 \times \mathrm{PS}$ & $-7.33 * *$ & $-18.99 * *$ & $-23.04 * *$ & -7.33 & $-18.99 * *$ & $-23.04 * *$ & $-7.40 * *$ & $-19.03 * *$ & $-23.08 * *$ \\
\hline 23 & $\mathrm{KM}-8 \times \mathrm{HM}$ & $61.41 * *$ & $35.93 * *$ & $29.13 * *$ & $61.41 * *$ & $35.93 * *$ & $29.13 * *$ & $61.45^{* *}$ & $35.91 * *$ & $29.11 * *$ \\
\hline
\end{tabular}


Int.J.Curr.Microbiol.App.Sci (2021) 10(02): 1534-1549

\begin{tabular}{|c|c|c|c|c|c|c|c|c|c|c|}
\hline 24 & $\mathrm{KM}-8 \times \mathrm{DK}$ & $69.21 * *$ & $42.11 * *$ & $35.22 * *$ & $69.21 * *$ & $42.11 * *$ & $35.00 * *$ & $69.20 * *$ & $42.09 * *$ & $34.98 * *$ \\
\hline 25 & KM-9 $\times$ PS & $-5.76 * *$ & $-17.62 * *$ & $-21.74 * *$ & -5.76 & $-17.62 * *$ & $-21.74 * *$ & $-5.79 * *$ & $-17.63 * *$ & $-21.75 * *$ \\
\hline 26 & KM-9 × HM & $-22.01 * *$ & $-34.32 * *$ & $-37.39 * *$ & $-22.01 * *$ & $-34.32 * *$ & $-37.61 * *$ & $-22.02 * *$ & $-34.35 * *$ & $-37.64 * *$ \\
\hline 27 & KM-9 × DK & -0.27 & $-16.25 * *$ & $-20.43^{* *}$ & -0.27 & $-16.25 * *$ & $-20.43^{* *}$ & -0.29 & $-16.27 * *$ & $-20.42 * *$ \\
\hline 28 & $\mathrm{KM}-10 \times \mathrm{PS}$ & $62.31 * *$ & $47.83^{* *}$ & $40.43 * *$ & $62.31 * *$ & $47.83 * *$ & $40.43 * *$ & $62.32 * *$ & $47.82^{* *}$ & $40.45^{* *}$ \\
\hline 29 & $\mathrm{KM}-10 \times \mathrm{HM}$ & $65.83 * *$ & $51.03 * *$ & $43.48 * *$ & $65.83 * *$ & $51.03 * *$ & $43.48 * *$ & $65.85 * *$ & $51.03 * *$ & $43.51 * *$ \\
\hline \multirow[t]{4}{*}{30} & $\mathrm{KM}-10 \times \mathrm{DK}$ & $59.30 * *$ & $45.08 * *$ & $37.83 * *$ & $59.30 * *$ & $45.08 * *$ & $37.83 * *$ & $59.34 * *$ & $45.10 * *$ & $37.87 * *$ \\
\hline & SEm \pm & 0.270 & 0.270 & 0.270 & 2.699 & 2.699 & 2.699 & 1.500 & 1.500 & 1.500 \\
\hline & $\mathrm{CD}$ at $5 \%$ & 0.77 & 0.77 & 0.77 & 7.70 & 7.70 & 7.70 & 4.28 & 4.28 & 4.28 \\
\hline & $\mathrm{CD}$ at $1 \%$ & 1.03 & 1.03 & 1.03 & 10.29 & 10.29 & 10.29 & 5.72 & 5.72 & 5.72 \\
\hline
\end{tabular}

*and** indicate significance of values at $\mathrm{p}=0.05$ and $\mathrm{p}=0.01$, respectively. BP, BTP and CC: Heterosis over better parent, best parent and commercial check (Madhuras), respectively. PS: Punjab Sunheri HM: Hara Madhu DK: Durga Kranti 
Table.4 Heterosis (\%) over better parent, best parent and commercial check for fruit shape index, flesh thickness and rind thickness in muskmelon

\begin{tabular}{|c|c|c|c|c|c|c|c|c|c|c|}
\hline \multirow[t]{2}{*}{ Sl. No. } & \multirow[t]{2}{*}{ Crosses } & \multicolumn{3}{|c|}{ Fruit shape index } & \multicolumn{3}{|c|}{ Flesh thickness } & \multicolumn{3}{|c|}{ Rind thickness } \\
\hline & & BP & BTP & $\mathrm{CC}$ & BP & BTP & $\mathrm{CC}$ & BP & BTP & $\mathrm{CC}$ \\
\hline 1 & $\mathrm{KM}-1 \times \mathrm{PS}$ & $-0.99 * *$ & 0.00 & $-9.91 * *$ & $10.81 * *$ & $8.13 * *$ & $11.63 * *$ & $-8.89 * *$ & $-6.82 * *$ & $-4.65^{* *}$ \\
\hline 2 & $\mathrm{KM}-1 \times \mathrm{HM}$ & $58.42 * *$ & $60.00 * *$ & $44.14 * *$ & $47.93 * *$ & $36.84 * *$ & $41.34 * *$ & $-8.89 * *$ & $-6.82 * *$ & $-4.65^{* *}$ \\
\hline 3 & $\mathrm{KM}-1 \times \mathrm{DK}$ & $52.48 * *$ & $54.00 * *$ & $38.74 * *$ & $79.73 * *$ & $59.33 * *$ & $64.60 * *$ & $-28.89 * *$ & $-27.27 * *$ & $-25.58 * *$ \\
\hline 4 & $\mathrm{KM}-2 \times \mathrm{PS}$ & $-10.71 * *$ & 0.00 & $-9.91 * *$ & $70.94 * *$ & $68.90 * *$ & $74.75 * *$ & $-31.82 * *$ & $-31.82 * *$ & $-30.23 * *$ \\
\hline 5 & KM-2 $\times$ HM & $11.61 * *$ & $25.00 * *$ & $12.61 * *$ & $-9.44 * *$ & $-10.53 * *$ & $-7.43 * *$ & 0.00 & 0.00 & $2.33^{* *}$ \\
\hline 6 & $\mathrm{KM}-2 \times \mathrm{DK}$ & $10.71 * *$ & $24.00 * *$ & $11.71 * *$ & $7.51 * *$ & $6.22 * *$ & $9.90 * *$ & $-15.91 * *$ & $-15.91 * *$ & $-13.95 * *$ \\
\hline 7 & $\mathrm{KM}-3 \times \mathrm{PS}$ & $-7.48 * *$ & $-1.00 * *$ & $-10.81 * *$ & $60.93 * *$ & $56.94 * *$ & $62.13 * *$ & $-32.61 * *$ & $-29.55^{* *}$ & $-27.91 * *$ \\
\hline 8 & KM-3 $\times$ HM & $17.76^{* *}$ & $26.00 * *$ & $13.51 * *$ & $13.07 * *$ & $7.66 * *$ & $11.39 * *$ & $-8.33 * *$ & 0.00 & $2.33 * *$ \\
\hline 9 & $\mathrm{KM}-3 \times \mathrm{DK}$ & $-6.54 * *$ & 0.00 & $-9.91 * *$ & $-32.91 * *$ & $-35.89 * *$ & $-33.91 * *$ & $6.25 * *$ & $15.91 * *$ & $18.60 * *$ \\
\hline 10 & $\mathrm{KM}-4 \times \mathrm{PS}$ & $15.45^{* *}$ & $27.00 * *$ & $14.41 * *$ & $29.98 * *$ & $26.79 * *$ & $30.94 * *$ & $-13.04 * *$ & $-9.09 * *$ & $-6.98 * *$ \\
\hline 11 & KM-4 × HM & $-14.55^{* *}$ & $-6.00 * *$ & $-15.32 * *$ & 0.00 & $-7.66^{* *}$ & $-4.46 * *$ & $10.87 * *$ & $15.91 * *$ & $18.60 * *$ \\
\hline 12 & $\mathrm{KM}-4 \times \mathrm{DK}$ & $16.36^{* *}$ & $28.00 * *$ & $15.32 * *$ & $87.30 * *$ & $66.03 * *$ & $71.53 * *$ & $-19.57 * *$ & $-15.91 * *$ & $-15.12 * *$ \\
\hline 13 & $\mathrm{KM}-5 \times \mathrm{PS}$ & $30.00 * *$ & $30.00 * *$ & $17.12 * *$ & $40.29 * *$ & $40.19 * *$ & $44.80 * *$ & $-2.17 * *$ & $2.27 * *$ & $4.65^{* *}$ \\
\hline 14 & KM-5 × HM & 0.00 & 0.00 & $-9.91 * *$ & $37.41 * *$ & $37.32 * *$ & $41.83 * *$ & $-28.00 * *$ & $-18.18 * *$ & $-16.28 * *$ \\
\hline 15 & $\mathrm{KM}-5 \times \mathrm{DK}$ & $35.00 * *$ & $35.00 * *$ & $21.62 * *$ & $46.04 * *$ & $45.93 * *$ & $50.74 * *$ & $-40.82 * *$ & $-34.09 * *$ & $-32.56 * *$ \\
\hline 16 & KM-6 × PS & $47.00 * *$ & $47.00 * *$ & $32.43 * *$ & $19.41 * *$ & $16.27 * *$ & $20.30 * *$ & $8.89 * *$ & $11.36 * *$ & $13.95 * *$ \\
\hline 17 & KM-6 × HM & $10.00 * *$ & $10.00 * *$ & $-0.90 * *$ & $-0.52 *$ & $-8.13 * *$ & $-4.95 * *$ & $-2.22 * *$ & 0.00 & $1.16^{* *}$ \\
\hline 18 & KM-6 × DK & $1.00 * *$ & $1.00 * *$ & $-9.01 * *$ & $2.16^{* * *}$ & $-9.57 * *$ & $-6.44 * *$ & $-2.22 * *$ & 0.00 & $1.16^{* *}$ \\
\hline 19 & $\mathrm{KM}-7 \times \mathrm{PS}$ & $0.99 * *$ & $2.00 * *$ & $-8.11 * *$ & $-18.18 * *$ & $-20.10 * *$ & $-17.57 * *$ & $8.70 * *$ & $13.64 * *$ & $16.28 * *$ \\
\hline \multirow[t]{2}{*}{ Sl. No. } & \multirow[t]{2}{*}{ Crosses } & \multicolumn{3}{|c|}{ Fruit shape index } & \multicolumn{3}{|c|}{ Flesh thickness } & \multicolumn{3}{|c|}{ Rind thickness } \\
\hline & & BP & BTP & $\mathrm{CC}$ & BP & BTP & $\mathbf{C C}$ & BP & BTP & $\mathbf{C C}$ \\
\hline 20 & $\mathrm{KM}-7 \times \mathrm{HM}$ & $10.89 * *$ & $12.00 * *$ & $0.90 * *$ & $49.74 * *$ & $40.67 * *$ & $45.30 * *$ & $20.83 * *$ & $31.82 * *$ & $34.88 * *$ \\
\hline 21 & $\mathrm{KM}-7 \times \mathrm{DK}$ & $3.96 * *$ & $5.00 * *$ & $-5.41 * *$ & $32.14 * *$ & $23.92 * *$ & $28.22 * *$ & $-10.42 * *$ & $-2.27 * *$ & 0.00 \\
\hline 22 & $\mathrm{KM}-8 \times \mathrm{PS}$ & $-12.26^{* *}$ & $-7.00 * *$ & $-16.22 * *$ & $-31.70 * *$ & $-33.49 * *$ & $-31.19 * *$ & $6.52 * *$ & $11.36^{* * *}$ & $13.95^{* *}$ \\
\hline 23 & $\mathrm{KM}-8 \times \mathrm{HM}$ & $-1.89 * *$ & $4.00 * *$ & $-6.31 * *$ & $72.54 * *$ & $59.33 * *$ & $64.85 * *$ & $-25.53 * *$ & $-20.45 * *$ & $-18.60 * *$ \\
\hline
\end{tabular}


Int.J.Curr.Microbiol.App.Sci (2021) 10(02): 1534-1549

\begin{tabular}{|c|c|c|c|c|c|c|c|c|c|c|}
\hline 24 & $\mathrm{KM}-8 \times \mathrm{DK}$ & $1.89 * *$ & $8.00 * *$ & $-2.70 * *$ & $40.53 * *$ & $27.75^{* *}$ & $32.18 * *$ & $19.15 * *$ & $27.27 * *$ & $30.23 * *$ \\
\hline 25 & $\mathrm{KM}-9 \times \mathrm{PS}$ & $7.77 * *$ & $11.00 * *$ & 0.00 & $38.08 * *$ & $34.45 * *$ & $39.11 * *$ & $10.87 * *$ & $15.91 * *$ & $17.44 * *$ \\
\hline 26 & KM-9 × HM & $-3.88 * *$ & $-1.00 * *$ & $-10.81 * *$ & $3.11 * *$ & $-4.78 * *$ & $-1.49 * *$ & $-10.87 * *$ & $-6.82 * *$ & $-5.81 * *$ \\
\hline 27 & $\mathrm{KM}-9 \times \mathrm{DK}$ & $-8.74 * *$ & $-6.00 * *$ & $-15.32 * *$ & $-26.58 * *$ & $-33.01 * *$ & $-30.94 * *$ & $6.52 * *$ & $11.36^{* *}$ & $12.79 * *$ \\
\hline 28 & $\mathrm{KM}-10 \times \mathrm{PS}$ & $16.07 * *$ & $30.00 * *$ & $17.12^{* *}$ & $61.67 * *$ & $57.42 * *$ & $62.87 * *$ & $-26.09 * *$ & $-22.73^{* *}$ & $-20.93 * *$ \\
\hline 29 & $\mathrm{KM}-10 \times \mathrm{HM}$ & $13.39 * *$ & $27.00 * *$ & $14.41 * *$ & $39.90 * *$ & $29.19 * *$ & $33.66 * *$ & $2.04 * *$ & $13.64 * *$ & $16.28 * *$ \\
\hline \multirow[t]{4}{*}{30} & $\mathrm{KM}-10 \times \mathrm{DK}$ & $-0.89 * *$ & $11.00 * *$ & 0.00 & $18.62 * *$ & $6.70 * *$ & $10.40 * *$ & $-20.41 * *$ & $-11.36 * *$ & $-10.47 * *$ \\
\hline & $\mathrm{SEm} \pm$ & 0.078 & 0.078 & 0.078 & 0.179 & 0.179 & 0.179 & 0.039 & 0.039 & 0.039 \\
\hline & $\mathrm{CD}$ at $5 \%$ & 0.22 & 0.22 & 0.22 & 0.51 & 0.51 & 0.51 & 0.11 & 0.11 & 0.11 \\
\hline & $\mathrm{CD}$ at $1 \%$ & 0.30 & 0.30 & 0.30 & 0.68 & 0.68 & 0.68 & 0.15 & 0.15 & 0.15 \\
\hline
\end{tabular}

*and** indicate significance of values at $\mathrm{p}=0.05$ and $\mathrm{p}=0.01$, respectively. BP, BTP and CC: Heterosis over better parent, best parent and commercial check (Madhuras), respectively. PS: Punjab Sunheri HM: Hara Madhu DK: Durga Kranti 
Table.5 Heterosis (\%) over better parent, best parent and commercial check for cavity length and cavity breadth in muskmelon

\begin{tabular}{|c|c|c|c|c|c|c|c|}
\hline \multirow{2}{*}{$\begin{array}{l}\text { Sl. } \\
\text { No. }\end{array}$} & \multirow[t]{2}{*}{ Crosses } & \multicolumn{3}{|c|}{ Cavity length } & \multicolumn{3}{|c|}{ Cavity breadth } \\
\hline & & BP & BTP & $\mathrm{CC}$ & BP & BTP & $\mathrm{CC}$ \\
\hline 1 & $\mathrm{KM}-1 \times \mathrm{PS}$ & $-6.99 * *$ & $-6.99 * *$ & $-3.93 * *$ & -0.17 & $6.98 * *$ & $-4.93 * *$ \\
\hline 2 & $\mathrm{KM}-1 \times \mathrm{HM}$ & $23.45 * *$ & $23.45 * *$ & $27.61 * *$ & $-13.24 * *$ & $-13.24 * *$ & $-22.97 * *$ \\
\hline 3 & $\mathrm{KM}-1 \times \mathrm{DK}$ & $12.89 * *$ & $12.89 * *$ & $16.69 * *$ & $5.34 * *$ & $12.88^{* *}$ & 0.24 \\
\hline 4 & KM- $2 \times$ PS & $-13.33 * *$ & $-5.12 * *$ & $-1.93 * *$ & $-10.76^{* *}$ & $6.80 * *$ & $-5.09 * *$ \\
\hline 5 & KM-2 $\times$ HM & $1.69 *$ & $11.96 * *$ & $15.65^{* *}$ & $9.12 * *$ & $9.12 * *$ & $-3.10^{* *}$ \\
\hline 6 & $\mathrm{KM}-2 \times \mathrm{DK}$ & $9.00 * *$ & $16.61 * *$ & $20.47 * *$ & 0.46 & $16.82 * *$ & $3.82 * *$ \\
\hline 7 & KM-3 $\times$ PS & 0.14 & $9.63 * *$ & $13.32 * *$ & $4.19 * *$ & $24.69 * *$ & $10.73 * *$ \\
\hline 8 & KM-3 $\times$ HM & $-8.60 * *$ & 0.62 & $3.93 * *$ & $-15.92 * *$ & $-15.92 * *$ & $-25.28 * *$ \\
\hline 9 & $\mathrm{KM}-3 \times \mathrm{DK}$ & $-36.72 * *$ & $-32.30 * *$ & $-30.10 * *$ & $-46.30 * *$ & $-37.57 * *$ & $-44.59 * *$ \\
\hline 10 & KM-4 $\times$ PS & $-25.39 * *$ & $-18.32 * *$ & $-15.65 * *$ & $-20.18 * *$ & $-4.47 * *$ & $-15.10 * *$ \\
\hline 11 & KM-4 × HM & $-14.53 * *$ & $-5.90 * *$ & $-2.81 * *$ & $10.55 * *$ & $10.55^{* *}$ & $-1.83 * *$ \\
\hline 12 & $\mathrm{KM}-4 \times \mathrm{DK}$ & $4.50 * *$ & $11.80 * *$ & $15.49 * *$ & $8.31 * *$ & $25.94 * *$ & $11.84 * *$ \\
\hline 13 & $\mathrm{KM}-5 \times \mathrm{PS}$ & $-3.83 * *$ & $5.28 * *$ & $8.75 * *$ & 0.30 & $20.04 * *$ & $6.68 * *$ \\
\hline 14 & KM-5 $\times$ HM & $-4.94 * *$ & $4.66^{* *}$ & $8.19 * *$ & $15.03 * *$ & $15.03 * *$ & $2.15^{* *}$ \\
\hline 15 & $\mathrm{KM}-5 \times \mathrm{DK}$ & $4.35 * *$ & $11.65 * *$ & $15.41 * *$ & $-17.23 * *$ & $-3.76 * *$ & $-14.47 * *$ \\
\hline 16 & KM-6 $\times$ PS & $14.04 * *$ & $24.84 * *$ & $28.97 * *$ & $-27.65 * *$ & $-13.42 * *$ & $-23.05 * *$ \\
\hline 17 & KM-6 × HM & $-11.00 * *$ & $-2.02 * *$ & $1.28 *$ & $-13.42 * *$ & $-13.42 * *$ & $-23.05 * *$ \\
\hline 18 & KM-6 × DK & $-41.80 * *$ & $-37.73 * *$ & $-35.63 * *$ & $-45.53 * *$ & $-36.67 * *$ & $-43.80 * *$ \\
\hline 19 & $\mathrm{KM}-7 \times \mathrm{PS}$ & $-29.84 * *$ & $-26.24 * *$ & $-23.76 * *$ & $-22.91 * *$ & $-22.36 * *$ & $-31.08 * *$ \\
\hline 20 & KM-7 × HM & $15.07 * *$ & $20.96 * *$ & $25.04 * *$ & $20.57 * *$ & $20.57 * *$ & $7.15 * *$ \\
\hline 21 & $\mathrm{KM}-7 \times \mathrm{DK}$ & 0.00 & $5.12 * *$ & $8.59 * *$ & $12.97 * *$ & $13.77 * *$ & 1.11 \\
\hline 22 & KM- $8 \times$ PS & $-6.61 * *$ & 0.93 & $4.33 * *$ & $4.63 * *$ & $13.24 * *$ & 0.56 \\
\hline 23 & KM-8 $\times$ HM & $-3.16^{* *}$ & $4.66 * *$ & $8.19 * *$ & $21.65 * *$ & $21.65 * *$ & $8.11 * *$ \\
\hline 24 & $\mathrm{KM}-8 \times \mathrm{DK}$ & $-11.61 * *$ & $-5.43 * *$ & $-2.25 * *$ & $-1.98 * *$ & $6.08 * *$ & $-5.72 * *$ \\
\hline 25 & $\mathrm{KM}-9 \times \mathrm{PS}$ & $9.54 * *$ & $19.41 * *$ & $23.43 * *$ & $16.17 * *$ & $19.50 * *$ & $6.12 * *$ \\
\hline 26 & KM-9 $\times$ HM & 0.28 & $9.32 * *$ & $12.92 * *$ & $10.38 * *$ & $10.38 * *$ & $-1.99 * *$ \\
\hline 27 & $\mathrm{KM}-9 \times \mathrm{DK}$ & $-28.88 * *$ & $-23.91 * *$ & $-21.43 * *$ & $-29.22 * *$ & $-27.19 * *$ & $-35.29 * *$ \\
\hline 28 & KM-10 $\times$ PS & $-5.67 * *$ & $3.26^{* *}$ & $6.74 * *$ & $-13.98 * *$ & $-7.51 * *$ & $-17.81 * *$ \\
\hline 29 & $\mathrm{KM}-10 \times \mathrm{HM}$ & $-14.81 * *$ & $-6.21 * *$ & $-3.05 * *$ & $-21.29 * *$ & $-21.29 * *$ & $-30.13 * *$ \\
\hline 30 & $\mathrm{KM}-10 \times \mathrm{DK}$ & $-1.89 * *$ & $4.97 * *$ & $8.51 * *$ & $-4.33 * *$ & $2.86^{* *}$ & $-8.66 * *$ \\
\hline & SEm \pm & 0.446 & 0.446 & 0.446 & 0.412 & 0.412 & 0.412 \\
\hline & $\mathrm{CD}$ at $5 \%$ & 1.27 & 1.27 & 1.27 & 1.17 & 1.17 & 1.17 \\
\hline & $\mathrm{CD}$ at $1 \%$ & 1.70 & 1.70 & 1.70 & 1.57 & 1.57 & 1.57 \\
\hline
\end{tabular}

*and $* *$ indicate significance of values at $\mathrm{p}=0.05$ and $\mathrm{p}=0.01$, respectively. BP, BTP and CC: Heterosis over better parent, best parent and commercial check (Madhuras), respectively, PS: Punjab Sunheri HM: Hara Madhu DK: Durga Kranti 
Table.6 Heterosis (\%) over better parent, best parent and commercial check for total soluble solids, total sugars and $\beta$-carotene content of fruit in muskmelon

\begin{tabular}{|c|c|c|c|c|c|c|c|c|c|c|}
\hline \multirow[t]{2}{*}{ Sl. No. } & \multirow[t]{2}{*}{ Crosses } & \multicolumn{3}{|c|}{ Total soluble solids } & \multicolumn{3}{|c|}{ Total sugars } & \multicolumn{3}{|c|}{$\beta$-carotene content } \\
\hline & & BP & BTP & $\mathrm{CC}$ & BP & BTP & $\mathrm{CC}$ & BP & BTP & $\mathrm{CC}$ \\
\hline 1 & $\mathrm{KM}-1 \times \mathrm{PS}$ & $-2.29 * *$ & $-2.24 * *$ & $11.70 * *$ & 0.96 & 0.96 & $11.47 * *$ & $58.15^{* *}$ & $58.15 * *$ & $60.77 * *$ \\
\hline 2 & $\mathrm{KM}-1 \times \mathrm{HM}$ & $23.86 * *$ & $19.41 * *$ & $36.52 * *$ & $18.37 * *$ & $14.07 * *$ & $25.96 * *$ & $56.96 * *$ & $50.74 * *$ & $53.24 * *$ \\
\hline 3 & $\mathrm{KM}-1 \times \mathrm{DK}$ & $-11.50 * *$ & $-16.71 * *$ & $-4.77 * *$ & $-5.64 * *$ & $-11.89 * *$ & $-2.72 * *$ & $51.37 * *$ & $45.37 * *$ & $47.78 * *$ \\
\hline 4 & $\mathrm{KM}-2 \times \mathrm{PS}$ & $49.24 * *$ & $49.29 * *$ & $70.61 * *$ & $35.24 * *$ & $35.25 * *$ & $49.36 * *$ & $55.16 * *$ & $55.17 * *$ & $57.74 * *$ \\
\hline 5 & $\mathrm{KM}-2 \times \mathrm{HM}$ & $22.94 * *$ & $18.59 * *$ & $35.51 * *$ & $23.48 * *$ & $18.99 * *$ & $31.40 * *$ & -17.39 & -22.15 & -20.86 \\
\hline 6 & $\mathrm{KM}-2 \times \mathrm{DK}$ & $40.81 * *$ & $32.59 * *$ & $51.51 * *$ & $10.40 * *$ & $3.01 * *$ & $13.81 * *$ & -15.66 & -20.53 & -19.21 \\
\hline 7 & $\mathrm{KM}-3 \times \mathrm{PS}$ & $46.47 * *$ & $46.47 * *$ & $67.45 * *$ & $47.95 * *$ & $47.95 * *$ & $63.47 * *$ & 24.84 & 24.84 & $26.91^{*}$ \\
\hline 8 & $\mathrm{KM}-3 \times \mathrm{HM}$ & $-12.45^{* *}$ & $-15.53 * *$ & $-3.50 * *$ & $-8.01 * *$ & $-11.34 * *$ & $-2.11 * *$ & 18.93 & -5.06 & -3.48 \\
\hline 9 & $\mathrm{KM}-3 \times \mathrm{DK}$ & $24.31 * *$ & $17.06 * *$ & $33.76 * *$ & $22.40 * *$ & $14.21 * *$ & $26.19 * *$ & -12.72 & -19.39 & -18.06 \\
\hline 10 & $\mathrm{KM}-4 \times \mathrm{PS}$ & $14.29 * *$ & $14.35 * *$ & $30.67 * *$ & 0.82 & 0.82 & $11.32 * *$ & 6.21 & 6.21 & 7.97 \\
\hline 11 & $\mathrm{KM}-4 \times \mathrm{HM}$ & $11.92 * *$ & $9.88 * *$ & $25.62 * *$ & $12.20 * *$ & $8.06 * *$ & $19.40 * *$ & $49.58 * *$ & 19.41 & 21.38 \\
\hline 12 & $\mathrm{KM}-4 \times \mathrm{DK}$ & $42.66 * *$ & $40.12 * *$ & $60.12 * *$ & $36.75^{* *}$ & $27.60 * *$ & $40.98 * *$ & -24.53 & $-30.30 *$ & $-29.15^{*}$ \\
\hline 13 & KM-5 × PS & -1.35 & -1.29 & $12.78 * *$ & $2.53 * *$ & $2.46 * *$ & $13.21 * *$ & 6.99 & 6.99 & 8.77 \\
\hline 14 & $\mathrm{KM}-5 \times \mathrm{HM}$ & $12.32 * *$ & $8.35^{* *}$ & $23.81 * *$ & $21.84 * *$ & $17.35^{* *}$ & $29.66 * *$ & 3.32 & -12.48 & -11.03 \\
\hline 15 & $\mathrm{KM}-5 \times \mathrm{DK}$ & $34.19 * *$ & $26.35 * *$ & $44.38 * *$ & $28.57 * *$ & $22.95 * *$ & $35.85^{* *}$ & $-28.85^{*}$ & $-34.29 * *$ & $-33.20 *$ \\
\hline 16 & KM-6 × PS & $3.47 * *$ & $3.53 * *$ & $18.29 * *$ & $-6.63 * *$ & $-6.69 * *$ & $3.09 * *$ & $39.18 * *$ & $39.18 * *$ & $41.49 * *$ \\
\hline 17 & KM-6 × HM & $43.20 * *$ & $38.12 * *$ & $57.83 * *$ & $35.60 * *$ & $30.60 * *$ & $44.30 * *$ & 17.84 & 6.62 & 8.39 \\
\hline 18 & KM-6 × DK & $18.56 * *$ & $14.24 * *$ & $30.60 * *$ & $14.71 * *$ & $7.10 * *$ & $18.26 * *$ & 24.00 & 14.51 & 16.41 \\
\hline 19 & KM-7 $\times$ PS & $22.59 * *$ & $22.59 * *$ & $40.15^{* *}$ & $19.75^{* *}$ & $19.67 * *$ & $32.23 * *$ & $-38.59 * *$ & $-38.59 * *$ & $-37.57 * *$ \\
\hline \multirow[t]{2}{*}{ Sl. No. } & \multirow[t]{2}{*}{ Crosses } & \multicolumn{3}{|c|}{ Total soluble solids } & \multicolumn{3}{|c|}{ Total sugars } & \multicolumn{3}{|c|}{$\beta$-carotene content } \\
\hline & & BP & BTP & $\mathrm{CC}$ & BP & BTP & $\mathrm{CC}$ & BP & BTP & $\mathrm{CC}$ \\
\hline 20 & $\mathrm{KM}-7 \times \mathrm{HM}$ & $-37.64 * *$ & $-39.88 * *$ & $-31.27 * *$ & $-40.99 * *$ & $-43.17 * *$ & $-37.21 * *$ & $-22.71 * *$ & $-29.85^{*}$ & $-28.69 *$ \\
\hline 21 & $\mathrm{KM}-7 \times \mathrm{DK}$ & $-12.31 * *$ & $-17.41 * *$ & $-5.65 * *$ & $-14.93 * *$ & $-20.63 * *$ & $-12.30 * *$ & $-16.99 *$ & -23.34 & -22.07 \\
\hline 22 & $\mathrm{KM}-8 \times \mathrm{PS}$ & $-26.47 * *$ & $-26.47 * *$ & $-15.94 * *$ & $-16.47 * *$ & $-16.53 * *$ & $-7.77 * *$ & 8.51 & 8.51 & 10.31 \\
\hline 23 & KM-8 × HM & -0.31 & $-3.88 * *$ & $9.89 * *$ & $-4.47 * *$ & $-7.92 * *$ & $1.66 * *$ & $33.09 *$ & 21.00 & 23.01 \\
\hline 24 & $\mathrm{KM}-8 \times \mathrm{DK}$ & $-14.31 * *$ & $-19.29 * *$ & $-7.80 * *$ & $-16.98 * *$ & $-22.54 * *$ & $-14.42 * *$ & $-27.67 *$ & $-33.20 *$ & $-32.10^{*}$ \\
\hline
\end{tabular}


Int.J.Curr.Microbiol.App.Sci (2021) 10(02): 1534-1549

\begin{tabular}{|c|c|c|c|c|c|c|c|c|c|c|}
\hline 25 & KM-9 $\times$ PS & $-40.88 * *$ & $-40.82 * *$ & $-32.41 * *$ & $-42.72 * *$ & $-42.76^{* *}$ & $-36.75 * *$ & $-29.68 *$ & $-29.68 *$ & $-28.51 *$ \\
\hline 26 & KM-9 × HM & $-13.36 * *$ & $-16.47 * *$ & $-4.51 * *$ & $-17.87 * *$ & $-20.90 * *$ & $-12.60 * *$ & -11.14 & -23.45 & -22.18 \\
\hline 27 & KM-9 × DK & $-21.62 * *$ & $-26.24 * *$ & $-15.67 * *$ & $-11.13 * *$ & $-17.08 * *$ & $-8.38 * *$ & $41.18 * *$ & $30.38 *$ & $32.54 *$ \\
\hline 28 & KM-10 $\times$ PS & $10.47 * *$ & $10.47 * *$ & $26.29 * *$ & $-8.82 * *$ & $-8.88 * *$ & 0.68 & 21.00 & 21.00 & 23.01 \\
\hline 29 & $\mathrm{KM}-10 \times \mathrm{HM}$ & $-17.63 * *$ & $-20.59 * *$ & $-9.21 * *$ & $-19.86 * *$ & $-22.81 * *$ & $-14.72 * *$ & $28.85^{*}$ & 18.69 & 20.66 \\
\hline \multirow[t]{4}{*}{30} & $\mathrm{KM}-10 \times \mathrm{DK}$ & $16.19 * *$ & $9.41 * *$ & $25.02 * *$ & $4.47 * *$ & $-2.46 * *$ & $7.70 * *$ & $68.37 * *$ & $55.49 * *$ & $58.07 * *$ \\
\hline & SEm \pm & 0.546 & 0.546 & 0.546 & 0.412 & 0.412 & 0.412 & 8.802 & 8.802 & 8.802 \\
\hline & $\mathrm{CD}$ at $5 \%$ & 1.56 & 1.56 & 1.56 & 1.17 & 1.17 & 1.17 & 25.10 & 25.10 & 25.10 \\
\hline & $\mathrm{CD}$ at $1 \%$ & 2.08 & 2.08 & 2.08 & 1.57 & 1.57 & 1.57 & 33.54 & 33.54 & 33.54 \\
\hline
\end{tabular}

*and** indicate significance of values at $\mathrm{p}=0.05$ and $\mathrm{p}=0.01$, respectively. BP, BTP and CC: Heterosis over better parent, best parent and commercial check

(Madhuras), respectively. PS: Punjab Sunheri HM: Hara Madhu DK : Durga Kranti 
The cross KM-2 x PS (74.75 \%) (Table 4) exhibited maximum standard heterosis for flesh thickness which was lower as comparable with that of reports by Vishwanatha (2003) (144.60\%) and which was higher as comparable with that reports by Subramanian (2008) (50.54\%) and it is attributed to use of different genetic stocks and also varied environmental conditions. Maximum negative (desirable) and significant heterosis over commercial check was observed in the cross KM-5 x DK (-32.56 \%) (Table 4) for rind thickness.

Reduction in cavity size has been considered as an important objective in muskmelon improvement and cavity length and cavity breadth reduction can reduce the cavity size and hence heterosis for cavity length and cavity breadth is desirable. The cross KM-6 $\mathrm{x}$ DK (-35.63 \%) (Table 5) exhibited maximum negative standard heterosis for cavity length and it is low as compared with the findings of Vishwanatha (2003) (-50.08\%). For cavity breadth the cross KM-3 x DK (-44.59 \%) (Table 5) exhibited maximum negative standard heterosis. Standard heterosis of 39.66 per cent had been reported by Vishwanatha (2003).

Total soluble solids of the fruit is an important quality trait where high TSS is preferred by consumers. The cross KM-2 x PS showed maximum heterosis of 70.61 per cent (Table 6) over commercial check which is very high as compared to 20.85 and 40.77 per cent reported by Vishwanatha (2003) and Shashikumar and Pitchaimuthu (2016), respectively and slightly higher as compared to 60.22 per cent noticed by Subramanian (2008). Total sugars are also an important quality character where consumers prefer sweet fruits. The cross KM-3 x PS exhibited maximum heterosis of 63.47 per cent (Table 6) over commercial check and is high as compared to 28.83 per cent reported by Moon et al., (2006). $\beta$-carotene content improves flesh colour appearance of the fruit and also an important nutritional quality parameter of the fruit. The cross KM-1 x PS exhibited maximum heterosis of 60.77 per cent (Table 6) over commercial check and which was higher as compared to 35.75 per cent had been reported by Moon et al., (2006) in muskmelon.

The main aim of any breeding programme is to enhance the yielding ability of the crop. Heterosis breeding offers quick and quantum jump in yield. On the basis of heterosis studies, it is concluded that the hybrids KM-2 $x$ PS, KM-1 x DK and KM-3 x PS were found to be superior hybrids selected for yield since, these crosses exhibited significant heterosis over commercial check for yield and also exhibited significant sca effects for yield per hectare. These crosses can be further assessed for their yield stability to confirm their potentiality and also their adaptability to different agro-climatic conditions before exploiting them on commercial scale.

\section{References}

Anonymous, 2014, Integrated handbook of horticulture (Kannada), Univ. Hort. Sci., Bagalkot. pp. 80-82.

Bayoumy, A. M., Mona, M., Yousry and Gamal, A. M. E., 2014, Combining ability and sensory evaluation for some hybrids of melon (Cucumis melo L.). Middle East J. Agric. Res., 3(3): 398411.

Chakrabarti, A. K., 2011, Importance of vegetables. In Textbook of Vegetables, Tuber Crops and Spices. Eds. Thamburaj, S. and Singh, N., Indian Council of Agric. Res., New Delhi, p. 4.

Choudhary, B. R., Dhoka, R. S. and Fagerio, M. S., 2003, Heterosis for yield and yield related attributes in muskmelon 
(Cucumis melo L.). Indian J. Genet., 63(1): 91-92.

Dhaliwal, M. S. and Lal, T., 1996, Genetics of some important characters using line xtester analysis in muskmelon. Indian J. Gen., 56(2): 207-213.

El-Shimi, A. Z. A., Mohamedein, S. A. and El-Fouly, A. H., 2003, Inheritance of some economic traits in melon (Cucumis melo L.). J. Agric. Sci. Mansoura Univ., 28(6): 4907-4918.

Hatem, M. K., Abd-Alla, A. M. and Shabrawy, R. A., 2009, Heterosis for yield components and some characters in melon (Cucumis melo L.). J. Agric. Res., 35(1): 101-106.

Kempthorne, O., 1957, An introduction to genetic statistics. John Wiley and Sons, New York, pp. 408-711.

Kesavan, P. K. and More, T. A., 1991, Use of monoecious lines in heterosis breeding in muskmelon (Cucumis melo L.). Veg. Sci., 18(1): 59-64.

Lal, T. and Singh, S., 1997, Genetic variability and selection indices in melons (Cucumis melo L.). Veg. Sci., 24(2): 111-117.

Moon, S. S., Munshi, A. D., Verma, V. K. and Sureja, A. K., 2006, Heterosis for biochemical traits in muskmelon
(Cucumis melo L.). Sabrao J. Breed. Genet., 38(1): 53-57.

Munshi, A. D. and Verma, V. K., 1997, Studies on heterosis in muskmelon (Cucumis melo L.). Veg. Sci., 24(2): 103-106.

Riggs, T. J., 1988, Breeding F1 hybrid varieties. J. Hort. Sci., 63: 362-369.

Shashikumar, K. T. and Pitchaimuthu, M., 2016, Heterosis and combining ability analysis of quantitative and qualitative traits in muskmelon (Cucumis melo L.). Int. J. Agric. Sci. Res., 6(2): 341348.

Singh, D., Nandapuri, K. S. and Sharma, B. R., 1976, Inheritance of some economic quantitative characters in an inter-varietal cross of muskmelon. $J$. Res., 13: 172-176.

Subramanian, D., 2008, Studies on heterosis expression and association of fruit yield and yield component characters among five intervarietal crosses of vellari melon (Cucumis melo L.) Madras Agric. J., 95(1-6): 24-31.

Vishwanatha, P. D., 2003, Genetic variability and heterosis studies in muskmelon (Cucumis melo L.). M. Sc. (Hort.) Thesis, Univ. Agric. Sci., Dharwad.

\section{How to cite this article:}

Shivaji Kallappa Duradundi, V. D. Gasti, Ravindra Mulage and Deelipkumar A. Masuthi. 2021. Heterosis Studies in Muskmelon (Cucumis melo L.) for Yield and Quality Traits. Int.J.Curr.Microbiol.App.Sci. 10(02): 1534-1549. doi: https://doi.org/10.20546/ijcmas.2021.1002.183 